\section{Tendências temporais de indicadores de atividade física e comportamento sedentário nas capitais da Região Sul do Brasil: 2006-2013}

Time trends of physical activity and sedentary behavior indicators in state capital of South region of Brazil: 2006-2013

Cassiano Ricardo Rech ${ }^{1}$

Rodrigo Siqueira Reis ${ }^{2,3}$

Adriano Akira Ferreira Hino ${ }^{2}$

Gisele Balbino Araujo Rodrigues de Sá4

Sheila Rizzato Stopa

Deborah Carvalho Malta ${ }^{6}$

\section{RESUMO}

O objetivo deste estudo foi analisar tendências temporais de atividade física (AF) e comportamento sedentário (CS) de acordo com indicadores sociodemográficos em adultos das capitais da Região Sul do Brasil, entre 2006 e 2013. Foram analisados dados de adultos ( $\geq 18$ anos), de Porto Alegre, Florianópolis e Curitiba, que participaram do VIGITEL. Foram analisadas tendências de $\mathrm{AF}$ no lazer, $\mathrm{AF}$ no deslocamento, inatividade física e comportamento sedentário (assistir TV). A prevalência de ativos no lazer, passou de 32,6\% no ano de 2009 para 38,3\% em 2013 ( $<<0,05)$. A variação de ativos do deslocamento foi estável entre 2009 e 2011, mas declinou de $16,4 \%$ para $11,0 \%$, entre 2011 e 2013 ( $\mathrm{p}<0,01$ ), especialmente entre as mulheres houve um declínio de 16,6\% em 2009 para 11,4\% em 2013 ( $\mathrm{p}<0,05)$. A proporção de inativos foi de $12,1 \%$ em 2006 e manteve-se em 13,7\% em 2013. O hábito de assistir TV diminui de $27,6 \%$ para $16,4 \%(\mathrm{p}<0,05)$ de 2006 para 2009. Já no período de 2010 a 2013 , a variação foi de $15,5 \%$ para $24,9 \%(\mathrm{p}<0,05)$. Os resultados apontam um aumento nas atividades físicas de lazer, diminuição dos ativos no deslocamento e estabilização dos inativos entre adultos das capitais da Região Sul do Brasil. A continuidade do monitoramento dos indicadores de atividade física e comportamento sedentário é fundamental para entender o processo temporal destes indicadores e estabelecer políticas e prioridades regionais a partir deste panorama epidemiológico.

\section{PALAVRAS-CHAVE}

Atividade física; Epidemiologia; Vigilância em Saúde Pública; Fatores de risco; Inquéritos epidemiológicos.

\footnotetext{
ABSTRACT

The aim of this study was to describe time trends in physical activity and sedentary time by sociodemographic indicators among adults $(18+$ years) residents in state Capitals of Brazilian South region. Data from 2006 to 2013 from the Surveillance System of Risk and Protective Factors for Chronic Diseases by telephone survey (VIGITEL) were analyzed limited to the state Capitals of the South region (Curitiba, Florianopolis, Porto Alegre). Trends of leisure time physical activity, walking or biking to/from work/school, prevalence of physical inactivity and the prevalence of adults reporting three or more hours per day watching television were described. Prevalence of physical activity in leisure time changed from $32.6 \%$ in 2009 to $38.3 \%$ in 2013 (p<.05). The variation on those who were active in transportation domain was stable between 2009 and 2011. But it declined from 16.4\% to 11.0\% between 2011 and 2013 (p<.01), especially among women, from 16.6\% in 2009 to $11.4 \%$ in 2013 (p<.05). The proportion of inactivity was $12.1 \%$ in 2006 and remained $13.7 \%$ in 2013. TV viewing decreased from $27.6 \%$ to $16.4 \%$ (p<.05) from 2006 to 2009. From 2010 to 2013, variation was from $15.5 \%$ to $24.9 \%(p<.05)$. The results show an increase in leisure physical activities, decreased number of active individuals in transportation and stabilization of the inactive among adults of southern Capitals in Brazil. Continued monitoring of indicators of physical activity and sedentary bebavior is essential to understand the temporal process of these indicators and establish policies and regional priorities.
}

Rev Bras Ativ Fís Saúde p. 47-56 DOI

http://dx.doi.org/10.12820/rbafs.v.20n1p47

1 Departamento de Educação Física. Centro de Desportos. Universidade Federal de Santa Catarina. Florianópolis. Santa Catarina. Brasil. crrechrahotmail.com

2 Grupo de Pesquisa em Atividade Física e Qualidade de Vida. Pontifícia Universidade Católica do Paraná. Curitiba. Paraná. Brasil. reis. rodrigolapucpr.br; akira.hinođapucpr.br

3.Departamento de Educação Física. Universidade Federal do Paraná. Curitiba. Paraná. Brasil

4 Programa de Pós Graduação em Planejamento Urbano. Pontifícia Universidade Católica do Paraná. Curitiba. Paraná. Brasil.

5 Departamento de Epidemiologia. Faculdade de Saúde Pública da Universidade de São Paulo. São Paulo. Brasil.

6 Universidade Federal de Minas Gerais. Minas Gerais. Brasil. dcmaltalauol.com.br

\section{KEYWORDS}

Physical activity; Epidemiology; Public health surveillance; Risk factors; Surveys. 


\section{INTRODUÇÃOO}

Monitorar os padrões de atividade física e sedentarismo na população é um importante processo para a prevenção e controle das doenças crônicas não transmissíveis ${ }^{1}$. No entanto, apenas nos anos recentes informações desta natureza tornaram-se disponíveis no Brasil ${ }^{2-7}$. Dados do Sistema de Vigilância de Fatores de Risco e Proteção para Doenças Crônicas por Inquérito Telefônico (VIGITEL), nas 26 capitais brasileiras e no Distrito Federal, entre 2006 e 2012, indicavam um aumento anual de 1,9\% na atividade física de lazer, especialmente entre os mais jovens e com maior escolaridade. Porém, apontavam uma tendência significativa de diminuição na proporção de indivíduos ativos no deslocamento e do hábito de assistir televisão ${ }^{8}$. Em outra análise secundária, com dados do VIGITEL, entre 2006 e 2012, em Porto Alegre, observou-se tendência de estabilização na atividade física de lazer, porém com um discreto aumento entre os homens, enquanto nas mulheres, no mesmo período, o índice declinou ${ }^{5}$. Em Pelotas/RS, coletas realizadas nos anos de 2002, 2007 e 2012 indicaram que a prevalência de inatividade física passou de 41,1\% para 54,4\% neste período, indicando um aumento significativo, exceto entre aqueles com elevada condição socioeconômica e mais velhos (>70 anos) ${ }^{6}$.

Observa-se que existem diferenças acerca da evolução dos níveis de atividade física em relação à localização geográfica e às características sociodemográficas, apesar das diferenças metodológicas dos estudos. Estas diferenças não têm sido adequadamente exploradas nos dados disponíveis para a população brasileira. Portanto, é necessário melhorar o entendimento sobre mudanças nas tendências de atividade física e comportamento sedentário em diferentes regiões e subgrupos populacionais, uma vez que existem importantes diferenças no padrão de morbimortalidade entre as regiões do país ${ }^{9}$. Por exemplo, apesar da mortalidade bruta causada pelas doenças crônicas ter aumento na última década, a mortalidade relativa diminuiu $20 \%$ no país. Contudo, na Região Sul do Brasil, houve um aumento da prevalência de diabetes e hipertensão, paralelo ao incremento do excesso de peso e esses aumentos podem estar associados com mudanças desfavoráveis na dieta, declínio da atividade física e aumento do comportamento sedentário ?.

Deste modo, este estudo tem como objetivo analisar a tendência temporal nos níveis de atividade física e comportamento sedentário, de acordo com indicadores sociodemográficos, em adultos das capitais da Região Sul, Brasil, no período de 2006 a 2013.

\section{MÉTODOS}

Os dados analisados neste estudo são provenientes do Sistema de Vigilância de Fatores de Risco e Proteção para Doenças Crônicas por Inquérito Telefônico (VIGITEL) dos anos de 2006 a 2013. O Vigitel é um inquérito telefônico de base populacional realizado, desde 2006, pelo Ministério da Saúde, nas 26 capitais brasileiras e no Distrito Federal. A população inclui adultos $(\geq 18$ anos), que possuam linha de telefone fixa em sua residência. Em cada capital, a amostragem foi realizada em duas etapas: (1) seleção randômica das linhas telefônicas residenciais fixas e (2) seleção aleatória do morador do domicílio a ser entrevistado. Foram entrevistadas aproximadamente 2000 pessoas em cada 
capital e no Distrito Federal, de modo a garantir um erro máximo de cerca de dois pontos percentuais para as estimativas de prevalência de qualquer fator de risco analisado. Neste estudo, foram analisados dados de oito inquéritos (2006 a 2013), das três capitais dos estados da Região Sul do Brasil (Curitiba, Florianópolis e Porto Alegre). Detalhes adicionais sobre o processo de amostragem estão disponíveis em publicações prévias ${ }^{10,11}$.

A prática de atividade física foi analisada nos domínios de lazer e deslocamento. No lazer, a atividade física foi mensurada com base em questões sobre a prática de exercício físico ou esporte nos últimos três meses e, em caso positivo, duração e frequência semanal dessa prática. O domínio do deslocamento foi avaliado por meio de questões sobre o uso de caminhada ou bicicleta para ir ou voltar do trabalho, sendo também computadas a duração e frequência semanal dessa prática. Outro fator analisado pelo VIGITEL é o comportamento sedentário, avaliado pelo tempo diário despendido assistindo televisão.

Para análise, os desfechos foram categorizados da seguinte maneira: (a) ativos no lazer: aqueles que praticam pelo menos 150 minutos semanais de atividade física de intensidade leve ou moderada ou pelo menos 75 minutos de atividade física de intensidade vigorosa. Para a construção deste indicador foram consideradas atividades de intensidade leve/moderada a caminhada, caminhada em esteira, musculação, hidroginástica, ginástica geral, natação, artes marciais, ciclismo e voleibol; corrida, corrida em esteira, ginástica aeróbica, futebol, basquetebol e tênis foram classificados como atividades de intensidade vigorosa. (b) ativos no deslocamento: fazem uso de caminhada ou bicicleta para ir e/ou voltar do trabalho por pelo menos 30 minutos por dia; (c) Fisicamente inativos: aqueles participantes que relataram não realizar esforços físicos intensos no trabalho, no ambiente doméstico e que não tenham se deslocado para o trabalho ou curso/escola caminhando ou de bicicleta perfazendo um mínimo de 10 minutos por trajeto por dia e que não praticaram qualquer atividade física no tempo livre nos últimos três meses; d) comportamento sedentário: aqueles que relataram mais de três horas por dia assistindo televisão. Os indicadores propostos são semelhantes aos indicadores utilizados em publicação prévia ${ }^{5,7}$, ${ }^{12}$. As questões do domínio de deslocamento sofreram modificações no ano de 2009, sendo adicionadas informações referentes ao deslocamento para a escola e/ou universidade, o que limita a comparação com anos anteriores.

As tendências temporais para cada indicador foram estratificadas por sexo (masculino; feminino), idade (18-24; 25-34; 35-44; 45-54; 55-64; 65+ anos) e escolaridade (0-4, 5-8, 9-11, 12+ anos). Houve modificação nas questões relacionadas com a atividade física de lazer e no deslocamento a partir do inquérito de 2009, deste modo, para estes indicadores as tendências foram analisadas no período entre 2009 a 2013. O percentual de mudança ao longo do tempo e a significância estatística das mudanças foram calculadas por meio de regressão linear, com cada indicador de atividade física como variável dependente e o período da análise como variável independente, adotando-se o nível de significância de $\mathrm{p}<0,05$. Todas as estimativas provenientes do VIGITEL são calculadas com a utilização de pesos pós-estratificação, objetivando igualar a composição sociodemográfica estimada para a população de adultos de cada capital. Estes pesos são compostos pelas variáveis: sexo, faixa etária e escolaridade; calculados pelo método "rake". Todas as análises foram realizadas no pacote estatístico Stata, versão 12.1 (Stata Corp., College Station, EUA). O estudo foi aprovado 
pela Comissão Nacional de Ética em Pesquisa em Seres Humanos (CONEP) $13081 / 2008$ e $355590 / 2013$. Por se tratar de entrevista por telefone, o termo de consentimento livre e esclarecido foi substituído pelo consentimento verbal obtido por ocasião dos contatos telefônicos com os entrevistados.

\section{RESULTADOS}

Os dados de 41.156 pessoas (53,9\% mulheres) estavam disponíveis no período incluído nas análises (2006-2013). A taxa de reposta mais baixa foi observada no ano de 2012 (62\%) em Florianópolis e Porto Alegre. Nas três capitais investigadas observou-se uma maior proporção de mulheres entrevistadas (Porto Alegre 54,8\%, Florianópolis 52,5\% e Curitiba 53,3\%).

As tendências temporais de atividade física e comportamento sedentário, em adultos das capitais da Região Sul do Brasil, no período de 2006 a 2013, são apresentados na Figura 1. No geral, a prevalência de ativos no lazer, na Região Sul, passou de 32,6\% no ano de 2009 para 38,3\% (p<0,05) em 2013. A variação de ativos do deslocamento foi estável entre 2009 e 2011, mas declinou de 16,4\% para 11,0\%, entre 2011 e 2013 (p<0,01). A proporção de inativos foi de 12,1\% em 2006 e manteve-se em 13,7\% em 2013. O hábito de assistir TV diminuiu de 27,6\% para 16,4\% (p<0,05) de 2006 para 2009. Já no período de 2010 a 2013, este indicador variou de $15,5 \%$ para $24,9 \%(\mathrm{p}<0,05)$.

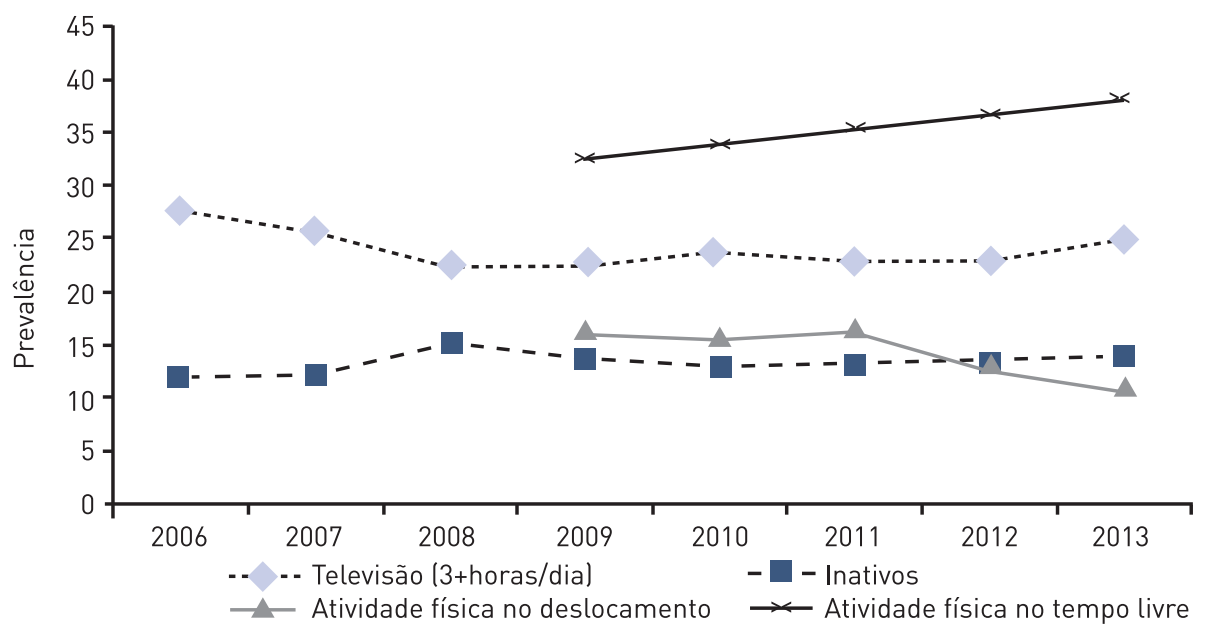

FIGURA 1 - Tendências temporais de atividade física e comportamento sedentário, nas capitais da Região Sul do Brasil, entre 2006 e 2013.

A tabela 1 mostra a tendência temporal de atividade física de lazer de acordo com sexo, faixa etária, anos de estudo e capital. Houve tendência de aumento na proporção de ativos no lazer em ambos os sexos, em adultos jovens e entre aqueles com 9 a 11 anos de estudo $(\mathrm{p}<0,05)$. $\mathrm{Na}$ análise das capitais, observou-se aumento significativo da proporção de ativos no lazer apenas em Porto Alegre (31,8\% em 2009 e 37,6\% em 2013; p<0,05) e Curitiba (31,4\% em 2009 e 37,4\% em 2013; p<0,05), com estabilização desta proporção em Florianópolis (40,7\% em 2009 e 43,9\% em 2013).

$\mathrm{O}$ número de mulheres ativas no deslocamento (tabela 2) declinou de 16,6\% em 2009 para 11,4\% em 2013 (p<0,05). Entre os homens não houve tendência significativa de mudança. Em relação à faixa etária observou-se es- 
TABELA 1 - Prevalência de ativos no lazer de acordo com sexo, faixa etária, escolaridade e cidade. Capitais da Região Sul, Brasil, 2009-2013.

\begin{tabular}{lcccccccc}
\hline & 2009 & 2010 & 2011 & 2012 & 2013 & Tendência & P \\
\hline Sexo & & & & & & & \\
\hline Masculino & 41,6 & 45,2 & 44,6 & 45,8 & 46,8 & $(+)$ & 0,045 \\
\hline $\begin{array}{l}\text { Feminino } \\
\text { Faixa etária }\end{array}$ & 24,9 & 24,1 & 27,6 & 29,1 & 31,0 & $(+)$ & 0,014 \\
\hline 18-24 & 47,2 & 49,2 & 50,9 & 54,0 & 53,3 & $(+)$ & 0,012 \\
\hline $25-34$ & 38,1 & 37,4 & 40,6 & 43,1 & 46,2 & $(+)$ & 0,012 \\
\hline $35-44$ & 28,1 & 29,7 & 33,5 & 32,3 & 32,7 & & 0,087 \\
\hline $45-54$ & 25,1 & 28,1 & 28,3 & 30,1 & 34,5 & $(+)$ & 0,012 \\
\hline $55-64$ & 25,7 & 30,6 & 31,1 & 31,0 & 32,0 & & 0,087 \\
\hline $65+$ & 26,7 & 24,4 & 22,9 & 25,7 & 25,1 & & 0,734 \\
\hline Escolaridade & & & & & & & \\
\hline 0-8 & 21,0 & 23,1 & 24,4 & 21,6 & 24,9 & & 0,298 \\
\hline $9-11$ & 33,5 & 35,4 & 38,0 & 42,1 & 40,5 & $(+)$ & 0,024 \\
\hline $12+$ & 45,6 & 44,8 & 44,3 & 45,9 & 48,9 & & 0,207 \\
\hline Cidades & & & & & & & \\
\hline Curitiba & 31,4 & 33,1 & 34,3 & 35,1 & 37,4 & $(++)$ & 0,001 \\
\hline Florianópolis & 40,7 & 37,1 & 42,9 & 43,1 & 43,9 & & 0,176 \\
\hline Porto Alegre & 31,8 & 33,8 & 34,6 & 37,0 & 37,6 & $(++)$ & 0,002 \\
\hline Ambos & 32,6 & 33,8 & 35,4 & 36,8 & 38,3 & $(++)$ & 0,001 \\
\hline
\end{tabular}

$(++)$ tendência de aumento significativo $(p<0,01) ;(+)$ tendência de aumento significativo $(p<0,05)$; $(--)$ tendência de diminuição significativa $(p<0,01)$; $(-)$ tendência de diminuição significativa $(p<0,05)$.

TABELA 2 - Prevalência de ativos no deslocamento de acordo com sexo, faixa etária, escolaridade e cidade. Capitais da Região Sul, Brasil, 2009-2013.

\begin{tabular}{|c|c|c|c|c|c|c|c|}
\hline Variáveis & 2009 & 2010 & 2011 & 2012 & 2013 & Tendência & $P$ \\
\hline \multicolumn{8}{|l|}{ Sexo } \\
\hline Masculino & 16,2 & 15,1 & 17,0 & 12,3 & 10,5 & & 0,088 \\
\hline Feminino & 16,6 & 15,9 & 15,8 & 13,6 & 11,4 & $(-)$ & 0,018 \\
\hline \multicolumn{8}{|l|}{ Faixa etária } \\
\hline $18-24$ & 22,5 & 20,6 & 21,8 & 15,3 & 15,7 & & 0,054 \\
\hline $25-34$ & 18,1 & 17,2 & 18,6 & 14,8 & 10,8 & & 0,075 \\
\hline $35-44$ & 19,1 & 20,1 & 18,9 & 13,6 & 12,9 & $(-)$ & 0,048 \\
\hline $45-54$ & 16,7 & 13,8 & 14,7 & 14,5 & 12,8 & & 0,118 \\
\hline $55-64$ & 10,9 & 10,4 & 14,0 & 10,8 & 8,4 & & 0,549 \\
\hline $65+$ & 4,9 & 5,7 & 5,9 & 5,7 & 3,0 & & 0,391 \\
\hline \multicolumn{8}{|l|}{ Escolaridade } \\
\hline $0-8$ & 17,7 & 16,0 & 17,4 & 14,2 & 10,7 & & 0,055 \\
\hline $9-11$ & 17,2 & 16,3 & 17,4 & 13,4 & 12,2 & & 0,058 \\
\hline $12+$ & 13,9 & 14,1 & 14,2 & 11,3 & 10,0 & & 0,056 \\
\hline \multicolumn{8}{|l|}{ Cidades } \\
\hline Curitiba & 17,4 & 14,9 & 15,9 & 13,8 & 10,5 & $(-)$ & 0,033 \\
\hline Florianópolis & 16,8 & 17,6 & 15,8 & 14,2 & 11,9 & $(-)$ & 0,026 \\
\hline Porto Alegre & 15,0 & 15,6 & 17,1 & 11,7 & 11,4 & & 0,187 \\
\hline Ambos & 16,4 & 15,5 & 16,4 & 13,0 & 11,0 & $(-)$ & 0,047 \\
\hline
\end{tabular}

$(++)$ tendência de aumento significativo $(p<0,01) ;(+)$ tendência de aumento significativo $(p<0,05)$; $(--)$ tendência de diminuição significativa $(p<0,01)$; (-) tendência de diminuição significativa $(p<0,05)$. 
tabilidade no número de ativos no deslocamento, exceto em adultos entre 35 e 44 anos, onde houve um declínio de 5,2 pontos percentuais $(\mathrm{p}<0,05)$, entre 2009 e 2013. Neste período, este declínio também foi observado em Curitiba (17,4\% em 2009 e 10,5\% em 2014; p<0,05) e Florianópolis (16,8\% em 2009 e $11,9 \%$ em $2013 ;$ p<0,05).

Em relação a prevalência de adultos inativos, não foi observada tendência significativa em relação à sexo, faixa etária, anos de estudo e cidade (tabela 3). Já o hábito de assistir televisão (tabela 4) apresentou tendência de declínio apenas entre adultos mais velhos (entre 55 e 64 anos) e com menos de 11 anos de estudo $(\mathrm{p}<0,05)$, mantendo-se estável nos demais subgrupos analisados.

TABELA 3 - Prevalência de inativos de acordo com sexo, faixa etária, escolaridade e cidade. Capitais da Região Sul, Brasil, 2006-2013.

\begin{tabular}{lcccccccccc}
\hline Variáveis & 2006 & 2007 & 2008 & 2009 & 2010 & 2011 & 2012 & 2013 & Tendência & $P$ \\
\hline Sexo & & & & & & & & & \\
\hline Masculino & 13,4 & 13,7 & 14,9 & 14,8 & 13,4 & 11,9 & 14,2 & 13,3 & 0,533 \\
\hline Feminino & 10,9 & 11,0 & 15,4 & 12,9 & 12,5 & 13,7 & 12,9 & 14,1 & 0,199 \\
\hline Faixa etária & & & & & & & & & 0,332 \\
\hline $18-24$ & 8,9 & 9,8 & 13,1 & 11,4 & 9,6 & 11,2 & 10,0 & 12,8 & 0,800 \\
\hline $25-34$ & 7,5 & 9,6 & 13,0 & 10,6 & 8,2 & 8,9 & 9,2 & 9,0 & 0,918 \\
\hline $35-44$ & 10,3 & 10,1 & 11,2 & 11,4 & 10,2 & 6,4 & 11,6 & 11,1 & 0,995 \\
\hline $45-54$ & 9,3 & 10,9 & 12,9 & 11,9 & 12,7 & 11,7 & 11,2 & 9,5 & 0,514 \\
\hline $55-64$ & 15,8 & 13,6 & 16,1 & 15,5 & 15,9 & 14,9 & 15,5 & 16,0 & 0,382 \\
\hline 65+ & 31,5 & 26,3 & 32,8 & 29,0 & 28,9 & 33,7 & 31,2 & 31,9 & \\
\hline Escolaridade & & & & & & & & & 0,095 \\
\hline 0-8 & 14,1 & 13,2 & 16,2 & 15,5 & 15,3 & 14,4 & 17,3 & 16,0 & 0,987 \\
\hline 9-11 & 10,1 & 10,6 & 12,6 & 12,2 & 10,8 & 10,3 & 11,1 & 10,9 & 0,692 \\
\hline $12+$ & 11,6 & 12,9 & 17,0 & 13,5 & 12,6 & 14,2 & 12,6 & 14,5 & \\
\hline Cidades & & & & & & & & & 0,700 \\
\hline Curitiba & 11,8 & 11,0 & 16,2 & 13,4 & 12,2 & 11,8 & 13,3 & 13,5 & 0,715 \\
\hline Florianópolis & 10,5 & 12,7 & 14,2 & 13,5 & 13,1 & 11,9 & 11,4 & 13,4 & 0,069 \\
\hline Porto Alegre & 12,9 & 13,6 & 14,2 & 14,3 & 13,7 & 14,5 & 14,5 & 14,0 & 13,7 \\
\hline Ambos & 12,1 & 12,2 & 15,2 & 13,7 & 12,9 & 12,9 & 13,5 & 13,7 \\
\hline
\end{tabular}

$(++)$ tendência de aumento significativo $(p<0,01) ;(+)$ tendência de aumento significativo $(p<0,05)$; $(--)$ tendência de diminuição significativa $(p<0,01)$; (-) tendência de diminuição significativa $(p<0,05)$.

TABELA 4 - Prevalência de adultos que relatam assistir televisão ( 33 horas/dia), de acordo com sexo, faixa etária, escolaridade e cidade. Capitais da Região Sul, Brasil, 2006-2013.

\begin{tabular}{lcccccccccc}
\hline $\begin{array}{l}\text { Variáveis } \\
\text { Sexo }\end{array}$ & 2006 & 2007 & 2008 & 2009 & 2010 & 2011 & 2012 & 2013 & Tendência & $P$ \\
\hline Masculino & 24,9 & 23,8 & 20,1 & 21,0 & 23,4 & 20,9 & 22,7 & 22,9 & \\
\hline Feminino & 30,0 & 27,1 & 24,5 & 24,5 & 23,7 & 24,0 & 22,8 & 26,5 & 0,533 \\
\hline Faixa etária & & & & & & & & & & 0,115 \\
\hline $18-24$ & 25,3 & 27,1 & 23,9 & 20,8 & 19,7 & 24,3 & 22,2 & 27,4 & \\
\hline $25-34$ & 24,3 & 22,3 & 18,5 & 22,6 & 23,7 & 21,5 & 23,6 & 25,3 & 0,811 \\
\hline $35-44$ & 25,0 & 23,9 & 18,7 & 20,0 & 19,5 & 22,1 & 20,1 & 20,4 & 0,424 \\
\hline $45-54$ & 27,7 & 24,5 & 21,5 & 21,1 & 23,1 & 19,7 & 22,1 & 22,5 & & 0,165 \\
\hline $55-64$ & 32,9 & 28,1 & 28,7 & 26,5 & 28,4 & 24,4 & 25,9 & 24,5 & $(--)$ & 0,007 \\
\hline $65+$ & 38,8 & 32,4 & 29,8 & 29,8 & 30,7 & 25,7 & 23,7 & 31,6 & & 0,064 \\
\hline
\end{tabular}




\begin{tabular}{lcccccccccc}
\hline $\begin{array}{l}\text { Variáveis } \\
\text { Escolaridade }\end{array}$ & 2006 & 2007 & 2008 & 2009 & 2010 & 2011 & 2012 & 2013 & Tendência & $P$ \\
\hline 0-8 & & & & & & & & & & \\
\hline 9-11 & 34,3 & 28,6 & 27 & 27,1 & 27,1 & 25,3 & 27,1 & 26,3 & $(-)$ & 0,042 \\
\hline $12+$ & 29,6 & 28,3 & 24,8 & 23,6 & 25,8 & 24,1 & 24,0 & 29,0 & 0,462 \\
\hline Cidades & 16,7 & 17,4 & 13,7 & 16,8 & 16,6 & 17,9 & 16,9 & 19 & 0,206 \\
\hline Curitiba & 25,1 & 23,9 & 21,1 & 21,1 & 21,8 & 19,4 & 19,6 & 22,3 & \\
\hline Florianópolis & 30,7 & 24,9 & 21,7 & 22,4 & 23,6 & 24,8 & 24,7 & 24,8 & 0,066 \\
\hline Porto Alegre & 29,8 & 27,8 & 24,2 & 25,2 & 25,6 & 25,8 & 26,0 & 28,1 & 0,407 \\
\hline Ambos & 27,6 & 25,6 & 22,4 & 22,8 & 23,5 & 22,6 & 22,8 & 24,9 & 0,551 \\
\hline
\end{tabular}

$(++)$ tendência de aumento significativo $(p<0,01) ;(+)$ tendência de aumento significativo $(p<0,05)$ $(--)$ tendência de diminuição significativa $(p<0,01) ;(-)$ tendência de diminuição significativa $(p<0,05)$

\section{DISCUSSÃO}

Evidências temporais sugerem alterações nos níveis de atividade física e comportamento sedentário nas capitais da Região Sul do Brasil entre 2006 e 2013. Houve um aumento nas atividades de lazer, com declínio nas atividades de deslocamento e estabilização na prevalência de inativos e do comportamento sedentário, expresso pelo tempo assistindo televisão. Florianópolis manteve-se durante todo o período da análise como a capital da Região Sul com a maior proporção de adultos ativos no lazer, porém, essa prevalência apresentou tendência de estabilização, diferente de Curitiba e Porto Alegre, onde houve aumento significativo no período analisado. Esses resultados são de grande importância para o monitoramento dos níveis de atividade física e comportamento sedentário no Brasil, especialmente por analisar uma região específica do país, que apresenta características socioculturais e econômicas distintas de outras regiões do país. Por exemplo, o índice de desenvolvimento humano (IDH) das capitais da Região Sul é considerado superior à média nacional (Curiti$\mathrm{ba}=0,823$; Florianópolis=0,847; Porto Alegre=0,805; média nacional=0,744) ${ }^{13}$.

A tendência de aumento nas atividades físicas de lazer é convergente com dados das 26 capitais do Brasil e do Distrito Federal entre 2006 e $2012{ }^{8}$ e, também é similar ao reportado em evidências internacionais ${ }^{1,14,15}$. Ainda é difícil explicar os reais motivos para esse aumento, porém, é possível que o crescente aumento da escolaridade e renda observado ao longo da última década ${ }^{16}$ e a implementação de políticas públicas de promoção da saúde possam ter contribuído para que um maior acesso de diferentes grupos populacionais aos benefícios da prática de atividade física, e a equipamentos e espaços de lazer para a prática de atividade física. Todavia, tais aspectos necessitam ser melhor compreendidos em estudos específicos sobre tais temas. Apesar deste aparente panorama positivo, existe a necessidade de manter e ampliar as políticas de estímulo para a atividade física de lazer, pois ainda aproximadamente $62 \%$ dos adultos em Porto Alegre e em Curitiba não atingem níveis recomendados de atividade física no lazer. Assim, continuar os investimentos no sistema de monitoramento da atividade física de lazer, bem como de outros fatores de proteção e risco para as DCNT, pode melhorar o entendimento deste impacto em desfechos de saúde.

Em relação à atividade física no deslocamento, o declínio observado nas capitais da Região Sul é similar ao relatado na média nacional ${ }^{8}$. A combinação 
de aspectos econômicos, infra-estrutura e sociais pode ter contribuído para estes resultados, particularmente, o aumento do poder de compra de veículos automotores. Por exemplo, entre 2001 e 2012 houve um aumento de 138\% na frota de veículos no Brasil, na Região Sul a frota de veículos passou de 5,2 milhões para 10,7 milhões, um crescimento de $105,7 \%{ }^{17}$. Além disso, a falta de estímulos para a inclusão de modais alternativos ao uso do carro/moto, como transportes públicos de qualidade, construção de ciclovias, estímulo fiscal para aquisição de bicicletas, maior segurança nas rotas de uso da bicicleta pode explicar a baixa adesão a modos ativos de transporte. O declínio da atividade física no deslocamento é um desafio nos próximos anos para a Região Sul, pois com a estabilização na tendência de atividade física de lazer, promover políticas para o deslocamento ativo deve estar na agenda da promoção da saúde, especialmente entre mulheres que apresentaram declínios mais acentuados de inatividade no deslocamento.

Outro importante achado deste estudo foi a estabilização da proporção de adultos inativos. Uma das razões para essa tendência pode estar relacionada com o desenvolvimento de políticas e ações do Ministério da Saúde que visam reduzir a prevalência de doenças crônicas não transmissíveis ${ }^{18}$. Além disso, a publicação da Política Nacional de Promoção da Saúde ${ }^{19}$, republicada em $2014^{20}$, evidenciou a prioridade da discussão do tema, o que resultou no desenvolvimento e avaliação de programas locais em diversas regiões do país, dentre elas, a Região Sul, com o CuritibAtiva ${ }^{21}$, tem como objetivo aumentar o acesso da população à prática de atividade física e pode ter auxiliado na estabilização do número de inativos da região. Além disso, observou-se um declínio do comportamento sedentário, expresso pelo tempo assistindo televisão, entre aqueles com menor escolaridade. Ainda que não seja possível apontar as causas diretas deste fenômeno, a combinação de aumento de renda, mesmo entre aqueles de menor escolaridade, pode ter contribuído por mudança nos hábitos de lazer, como por exemplo, maior uso de telas ligadas à internet. De fato, houve um aumento substancial na aquisição de computadores portáteis, telefones celulares e acesso à internet ao longo da última década ${ }^{16}$. Esta mudança pode indicar uma mudança no tipo de atividade sedentária com menor tempo de televisão e maior tempo em outras telas ou internet.

Os achados deste estudo confirmam que há uma mudança em curso tanto nos níveis quanto no perfil das atividades físicas entre os adultos das capitais do Sul do Brasil. O monitoramento desta mudança indica que há, ainda, necessidade de avançar de maneira substancial nas políticas de promoção de atividade física. Apesar do inequívoco avanço na prática de atividade física no lazer, é evidente que a redução dos níveis de inatividade requer ações amplas na comunidade, em especial nos modos de deslocamento e no planejamento das cidades. Além disto, a compreensão sobre novos hábitos de lazer que contribuam para novos indicadores sobre o tempo sedentário é desejável.

Algumas limitações devem ser consideradas neste estudo. A seleção dos indivíduos foi restrita àqueles com linha telefônica fixa. Contudo, ressalta-se que as capitais da Região Sul possuem elevados percentuais de cobertura telefônica. O comportamento sedentário foi mensurado a partir de um único indicador, o número de horas assistindo TV, o que pode subestimar o padrão deste comportamento. Porém, assistir TV é uma das atividades sedentárias mais comum entre os brasileiros o que possibilita uma análise de sua tendência temporal. 
De maneira semelhante ao que foi relatado na análise em conjunto com as 26 capitais brasileiras e o Distrito Federal, nas capitais da Região Sul do Brasil existe uma tendência de aumento da proporção de ativos no lazer. Também se observa uma estabilização nas prevalências de inativos e no hábito de assistir televisão. Porém, uma diminuição no deslocamento ativo. Este estudo analisa um amplo período e fornece uma análise específica das capitais da Região Sul do país. A continuidade do monitoramento dos indicadores de atividade física e comportamento sedentário é fundamental para que possamos entender o processo temporal destes indicadores e estabelecer políticas e prioridades regionais a partir deste panorama epidemiológico.

\section{REFERÊNCIAS}

1. Knuth AG, Hallal PC. Temporal trends in physical activity: a systematic review. J Phys Act Health. 2009;6(5):548-59.

2. Knuth AG, Bacchieri G, Victora CG, Hallal PC. Changes in physical activity among Brazilian adults over a 5-year period.J Epidemiol Community Health. 2010;64(7):591-5.

3. Hallal P, Knuth A, Reis R, Rombaldi A, Iser B, Bernal R. Tendências temporais de atividade física no Brasil. Rev Bras Epidemiol. 2011;14(1):53-60.

4. Matsudo V, Matsudo SM, Araújo TL, Andrade DR, Oliveira LC, Hallal PC. Time trends in physical activity in the state of Sao Paulo, Brazil: 2002-2008. Medicine and Science in Sports and Exercise. 2010;42(12):2231-6.

5. Souza A, Silva S, Mielke G, Hallal P. Tendências temporais de atividade física e comportamento sedentário em Porto Alegre, Brasil: 2006-2012. Revista Brasileira de Atividade Física \& Saúde. 2014;19(4):514.

6. Hallal P, Cordeira K, Knuth A, Mielke G, Victora C. Ten-Year Trends in Total Physical Activity Practice in Brazilian Adults: 2002-2012. Journal of Physical Activity \& Health. 2014 (no prelo).

7. Mielke GI, Hallal PC, Malta DC, Lee I-M. Time trends of physical activity and television viewing time in Brazil: 2006-2012. International Journal of Behavioral Nutrition and Physical Activity. 2014;11(1):101.

8. Mielke GI, Hallal PC, Malta DC, Lee I-M. Time trends of physical activity and television viewing time in Brazil: 2006-2012. International Journal of Behavioral Nutrition and Physical Activity. 2014;11(1):101.

9. Schmidt MI, Duncan BB, Menezes AM, Monteiro CA, Barreto SM, Chor D, et al. Chronic non-communicable diseases in Brazil: burden and current challenges. The Lancet. 2011;377(9781):1949-61.

10. Moura EC, Morais Neto OL, Malta DC, Moura L, Silva NN, Bernal R, et al. Vigilância de fatores de risco para doenças crônicas por inquérito telefônico nas capitais dos 26 estados brasileiros e no Distrito Federal (2006). Revista Brasileira de Epidemiologia. 2008;11:20-37.

11. Malta DC, Moura EC, Castro AM, Cruz DKA, Neto OLM, Monteiro CA. Padrão de atividade física em adultos brasileiros: resultados de um inquérito por entrevistas telefônicas, 2006. Epidemiol Serv Saúde. 2009;18(1):7-16.

12. Florindo AA, Hallal PC, Moura ECd, Malta DC. Prática de atividades físicas e fatores associados em adultos, Brasil, 2006. Revista de Saúde Pública. 2009;43:65-73.

13. PNUD. Relatório do Desenvolvimento Humano 2014 - Sustentar o progresso humano: reduzir as vulnerabilidades e reforçar a resilência. 2014.

14. Stamatakis E, Ekelund U, Wareham NJ. Temporal trends in physical activity in England: the Health Survey for England 1991 to 2004. Preventive medicine. 2007;45(6):416-23.

15. Juneau C-E, Potvin L. Trends in leisure-, transport-, and work-related physical activity in Canada 1994,Äì2005. Preventive medicine. 2010;51(5):384-6.

16. IPEA. Desigualdades de renda no Brasil: uma análise da queda recente. IPEA, 2007.

17. INCT. Evolução da frota de automóveis e motos no Brasil 2001-2012. Rio de Janeiro: Instituto Nacional de Ciência e Tecnologia; 2013. 
18. Brasil. Plano de ações estratégicas para o enfrentamento das doenças crônicas não transmissíveis (DCNT) no Brasil 2011-2012.: Ministério da Saúde. Secretaria de Vigilância em Saúde. Departamento de Análise da Situação de Saúde.; 2011.

19. Brasil. Política nacional de promoção da saúde.: Ministério da Saúde. Secretaria de vigilânica em Saúde.2006.

20. Brasil. Redefine a Política Nacional de Promoção da Saúde. Brasilia: Ministério da Saúde. Secretaria de vigilânica em Saúde. 2014.

21. Hallal PC, Reis RS, Hino AAF, Santos MS, Grande D, Krempel M, et al. Avaliação de programas comunitários de promoção da atividade física: O caso de Curitiba, Paraná. Rev Bras Ativ Fís Saúde. 2009;14(2):104-14.

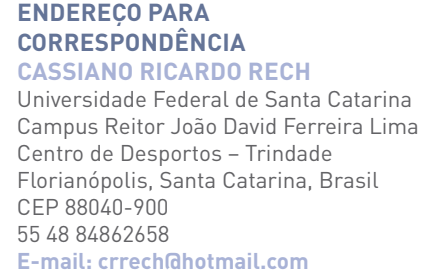

